\title{
Efficacy of a Pocket Pulse Oximeter and a Digital Pulse Oximeter as Screening Tools for Type 2 Diabetes Patients with Asymptomatic Lower Extremity Arterial Disease
}

Shivraj S HANCHINAL a , Avinash B SHIVANAL ${ }^{b}$, Supreet KAMNI ${ }^{c}$, Sneha SHEELWANTH ${ }^{\text {d, }}$ Vijay Prasad SANGISHETTI ${ }^{\mathrm{e}}$, Ambadasu BHARATHFe

aDepartment of General Medicine, Mahavir Institute of Medical College, Vikarabad, India

bMedical and Scientific Services, IQVIA, Bangalore, Karnataka, India

clinical Development, Novartis, Hyderabad, India

dESIC Medical College, Kalaburgi, India

eDepartment of Pharmacology, Govt Medical College, Shivpuri, Madhya Pradesh, India

fFaculty of Medical Sciences, University of the West Indies, Cave Hill, Barbados,

West Indies

ABSTRACT
Introduction: The risk of developing lower extremity arterial diseases (LEAD) increases by 2-4 folds
in patients with diabetes mellitus. Diabetic individuals with LEAD are more likely than non-diabetic
individuals to have limb amputations, and they have a higher risk of cardiovascular and cerebrovascular
problems. The present study sought to assess the efficacy of two pulse oximeters, a high-quality standard
digital pulse oximeter and an inexpensive pocket pulse oximeter, to detect LEAD in patients with diabetes
mellitus.
Material and methods: Qualifying patients were examined with a pocket pulse oximeter and digital
pulse oximeter for measuring SpO. of their index fingers, big toes in the supine position, and at 12-inch
elevation. All patients underwent Doppler waveform analysis examination of their lower extremity arteries
by an investigator, who was unaware of the pulse oximetry results. Doppler waveform analysis is used as
the standard. Sensitivity, specificity, the positive and negative predictive values were compared.
Results: Twenty nine of 100 legs examined had a significant LEAD, i.e., monophasic waveform by
Doppler waveform analysis. Pocket pulse oximetry showed a sensitivity of $72 \%$, a specificity of $98 \%$ and a
positive predictive value of $95 \%$, and a negative predictive value of $90 \%$. Digital pulse oximetry showed a
sensitivity of $83 \%, 98 \%$, a positive predictive value of $96 \%$, and a negative predictive value of $93 \%$.

Address for correspondence:

Dr. Ambadasu Bharatha, Lecturer in Pharmacology

Faculty of Medical Sciences, University of the West Indies, Cave Hill, Barbados, West Indies

Email: ambadasu.bharatha@cavehill.uwi.edu 
Conclusion: Pulse oximetry may be a useful additional tool to screen for LEAD in patients with diabetes mellitus. It has good sensitivity and specificity in detecting LEAD even in the asymptomatic phase, especially in type 2 diabetes mellitus patients. In comparison between two oximeters, digital pulse oximetry showed a sensitivity, and the higher sensitivity is desirable in a screening test.

Keywords: pocket pulse oximeter, $\mathrm{SpO}_{2}$, Doppler waveform analysis, digital pulse oximeter, type $2 \mathrm{DM}$, LEAD.

\section{INTRODUCTION}

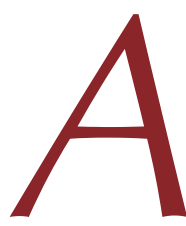
cute limb ischemias are either thrombotic or embolic (1). Chronic ischemia is primarily due to atherosclerotic changes in the lower extremity arteries, resulting from asymptomatic disease to limb-threatening gangrene (2). Lower extremity arterial disease (LEAD)/peripheral artery disease (PAD) is often one of the first signs of generalized atherosclerotic disease. Patients with intermittent claudication (IC) or critical limb ischemia (CLI) often have other atherosclerotic manifestations as well. Risk factors for LEAD are diabetes mellitus (DM), smoking, hypertension, hyperlipidemia, impaired fibrinolysis, inflammation, age, and genetic predisposition as similar to those for other manifestations of atherosclerosis, i.e., coronary artery disease and stroke (3).

The risk of developing PAD/LEAD increases by 2-4 folds in patients with diabetes mellitus (DM). Diabetic patients with LEAD had a higher risk of limb amputation and cardiovascular and cerebrovascular problems than non-diabetic patients (4).

The duration and severity of diabetes correlate with the incidence and extent of PAD. The data showed a higher prevalence of PAD in those with a longer duration of diabetes. The degree of diabetic control is an independent risk factor for PAD; with every $1 \%$ increase in $\mathrm{HbA} 1 \mathrm{c}$, the risk of PAD has been shown to increase by $28 \%$ (5).

The relation between asymptomatic and symptomatic LEAD is approximately $4: 1$. Interestingly, patients with asymptomatic LEAD have a similar increase in cardiovascular risk as those with intermittent claudication (6).

Peripheral vascular disease affects approximately 12 million people in the US (7). However, an accurate assessment of PAD prevalence in the diabetic population is confounded by various factors. Peripheral neuropathy in DM may alter pain perception; the condition often remained asymptomatic in studies using the ankle-brachial index $(A B I)$, which has been the preferred screening technique, the prevalence of PAD (defined as $A B I<0.90)$ in diabetic individuals ranging from $20 \%$ to $30 \%$ (7). In the Indian subcontinent, diabetes forms the largest group of patients with atherosclerotic PAD. Eighty million people $(8-10 \%)$ of the Indian population over the age of 55 years suffer from PAD (8).

It is preferable to diagnose LEAD before symptoms appear in diabetic people. It can better control risk factors, reduce morbidity, mortality, and implement an effective treatment for arterial disease associated with LEAD and cardiovascular patients.

Waveform analysis and duplex colour mapping are accurate Doppler ultrasound procedures. However, they are too expensive and ineffective for screening. On the other hand, although angiography studies are the gold standard, they are invasive and expensive; therefore, compliance among asymptomatic patients is poor, which makes it inappropriate for screening purposes (9).

Pulse oximeters are widely available and easy to use. As a result, pulse oximetry has been developed as a non-invasive screening method. The rationale for using fingertip pulse oximetry as screening for PAD is based on the hypothesis that there would be a gradient of oxygen saturation between upper and lower limbs in patients with significant arterial perfusion defects (10).

Therefore, the present study sought to assess the efficacy of two pulse oximeters, a high-quality standard digital pulse oximeter (DPO) and an inexpensive pocket pulse oximeter (PPO), to detect LEAD in patients with DM.

\section{METHODOLOGY}

tudy design: analytical, cross-sectional hospital-based study

Ethics approval: The study was authorised by the Institutional Ethics Committee, which fol- 
lowed the Declaration of Helsinki, a code of research ethics (HKES/MRMC/IEC/181045).

Study location: Basaweshwara teaching and General Hospital Kalaburagi, Karnataka, India

Study duration: two years

Source of the data: type 2 diabetes mellitus (T2DM) patients attending the out-patient clinic and admitted as inpatients

Sample size: Fifty patients

Inclusion criteria: patients of both sexes aged $\geq 40$ years with established T2DM and without any symptoms of lower extremity arterial disease

Exclusion criteria: painful inflammatory processes or extreme edema, revascularization procedures, or amputation over any fingers or toes.

Method: The study objectives were met by using a proforma to collect data: a complete history, general physical and systemic examination and any necessary investigations. Patients who satisfied the criteria had their $\mathrm{SpO}_{2}$ measured using a PPO and a DPO in the supine position and at a 12-inch elevation (11). All patients underwent a Doppler waveform analysis examination of their lower limb arteries (femoral, popliteal, tibial, posterior tibial, and dorsalis pedis arteries) by an investigator who was uninformed of the pulse oximetry data at a later time in the radiology department. Abnormal pulse oximetry of the toes was characterised as $\mathrm{SaO}_{2}$ value of more than $2 \%$ lower than the finger value or a reduction of more than $2 \%$ on an elevation of the leg (reduction from the value at the supine position) (decrease from the value at the supine position). Significant LEAD was defined as monophasic waveforms at any one of the lower extremity arteries during Doppler waveform analysis.

The benchmark for comparison is Doppler waveform analysis. When compared to arteriography, this test has a $97 \%$ specificity in the diagnosis of LEAD and has been utilised as the gold standard in prior research.

\section{RESULTS}

\section{ex and age distribution}

The majority of patients were males $36(72 \%)$ when compared to females 14 (28\%). The male:female ratio was 2.6:1 (Figure 1). Most patients were in the age group of 50-59 years (34\%)

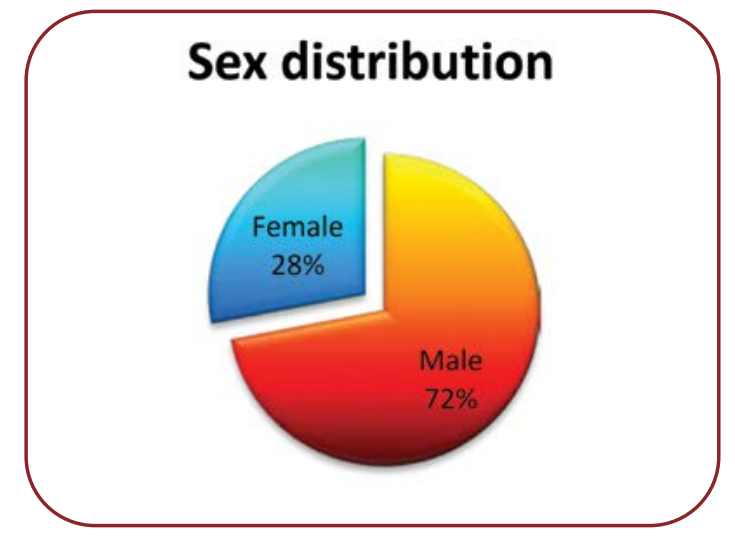

FIGURE 1. Sex distribution

\begin{tabular}{|c|c|c|}
\hline Age & No. of cases & Percent \\
\hline $40-49$ & 12 & 24.0 \\
\hline $50-59$ & 17 & 34.0 \\
\hline $60-69$ & 15 & 30.0 \\
\hline $70-79$ & 6 & 12.0 \\
\hline Total & $\mathbf{5 0}$ & $\mathbf{1 0 0 . 0}$ \\
\hline
\end{tabular}

\begin{tabular}{|l|l|}
\hline \multicolumn{2}{|l|}{ Age } \\
\hline Mean \pm SD & $56.2 \pm 10.1$ \\
\hline Range & 37.00 \\
\hline Minimum & 41.00 \\
\hline Maximum & 78.00 \\
\hline
\end{tabular}

TABLE 1. Age distribution

and $60-69$ years (30\%). The mean age was found to be $56.24+/-10.14$ years with a range from 41-78 years (Table 1 ).

\section{Duration of diabetes mellitus}

The known duration of diabetes ranged from one year to 21 years. The mean duration of DM was 9.45 years (Figure 2).

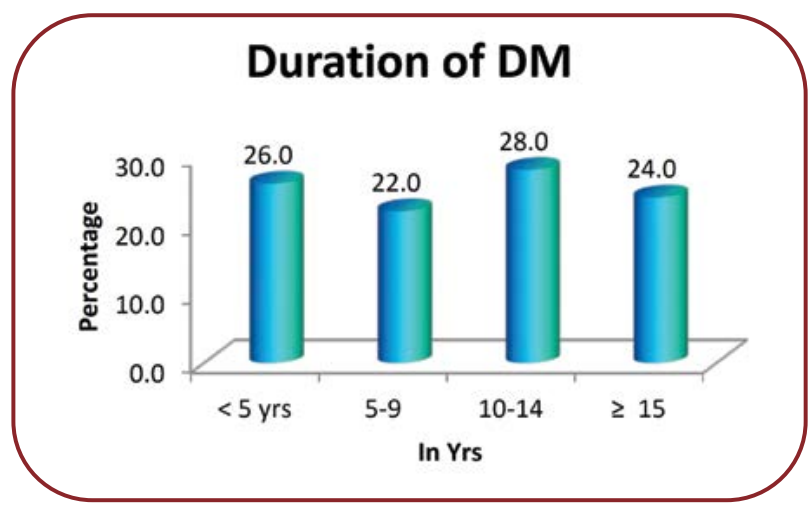

FIGURE 2. Duration of diabetes mellitus in patients screened for LEAD 
TA B LE 2. Relation between duration of diabetes and Doppler positivity for LEAD

\begin{tabular}{|l|l|l|l|}
\hline Duration of DM* in years & No. of cases & Doppler positive lead & Percentage \\
\hline$<5$ & 13 & 1 & $7.7 \%$ \\
\hline $5-9$ & 11 & 2 & $18.2 \%$ \\
\hline $10-14$ & 14 & 7 & $50 \%$ \\
\hline 15 and above & 12 & 9 & $75 \%$ \\
\hline
\end{tabular}

$* \mathrm{DM}=$ diabetes mellitus

TA B LE 3. Relation between duration of diabetes and Doppler positivity for LEAD

\begin{tabular}{|l|c|c|c|c|}
\hline Comorbidities & Males & Females & Total & Percentage \\
\hline Hypertension & 11 & 08 & 19 & $38 \%$ \\
\hline CAD* & 08 & 06 & 14 & $28 \%$ \\
\hline CVA $^{* *}$ & 03 & 00 & 03 & $06 \%$ \\
\hline Smoking history & 25 & 00 & 25 & $50 \%$ \\
\hline
\end{tabular}

*CAD $=$ coronary artery disease

$* * \mathrm{CVA}=$ cerebrovascular accident

TA B LE 4. Test results for LEAD by Doppler analysis, pocket pulse oximetry and digital pulse oximetry

\begin{tabular}{|l|l|l|l|l|l|}
\hline \multirow{2}{*}{ Test results } & \multirow{2}{*}{ No of tested limbs } & \multicolumn{2}{l|}{ Positives } & \multicolumn{2}{l|}{ Negatives } \\
\cline { 3 - 6 } & & True & False & True & False \\
\hline Doppler & 100 & 29 & 00 & 71 & 00 \\
\hline Pocket pulse oximetry & 100 & 21 & 01 & 70 & 08 \\
\hline Digital pulse oximetry & 100 & 24 & 01 & 70 & 05 \\
\hline
\end{tabular}

TABLE 5. Efficacy of pocket pulse oximetry and digital pulse oximetry in detecting LEAD in asymptomatic T2DM patients

\begin{tabular}{|l|l|l|}
\hline Oximetry & Pocket pulse oximetry & Digital pulse oximetry \\
\hline Sensitivity & $72 \%$ & $83 \%$ \\
\hline Specificity & $98 \%$ & $98 \%$ \\
\hline PPV* & $95 \%$ & $96 \%$ \\
\hline NPV** & $90 \%$ & $93 \%$ \\
\hline Accuracy & $91 \%$ & $94 \%$ \\
\hline
\end{tabular}

*PPV=positive predictive value

**NPV=negative predictive value

Relation between duration of diabetes and Doppler positivity for LEAD

The occurrence of LEAD increased as the duration of diabetes increased from $7.7 \%$ in patients with a duration of diabetes under $<$ five years to $75 \%$ in those with a duration of diabetes $>15$ years (Table 2 ).

\section{Selected comorbidities in type $\mathbf{2}$ diabetes mellitus patients screened for LEAD}

Out of 50 patients in the study, 19 (38\%) subjects had hypertension, 14 (28\%) coronary artery disease (CAD) and 3 (6\%) cerebrovascular accident (CVA), and 25 (50\%) patients (males only) had a smoking history (Table 3).

In our study, LEAD was detected by Doppler waveform analysis in 29 (29\%) of 100 studied limbs. Pocket pulse oximetry was truly positive in $21(21 \%)$ of tested limbs, falsely positive in one $(1 \%)$, truly negative in $70(70 \%)$ and falsely negative in eight (8\%). Digital pulse oximetry was truly positive in $24(24 \%)$ of tested limbs, falsely positive in one $(1 \%)$, truly negative in $70(70 \%)$ and falsely negative in five (5\%) (Table 4$)$.

The results of pocket pulse oximetry and digital pulse oximetry were compared with the standard Doppler waveform analysis to assess their efficacy. In our study, pocket pulse oximetry showed a sensitivity of $72 \%$, a specificity of $98 \%$, a positive predictive value (PPV) of $95 \%$, and a negative predictive value (NPV) of $90 \%$, and it was found to have an accuracy of $91 \%$. Digital pulse oximetry had a sensitivity of $83 \%$, a specificity of $98 \%$, a PPV of $96 \%$ and a NPV of $93 \%$, and it showed an accuracy of $94 \%$ (Table 5).

\section{DISCUSSION}

n our study, patients were aged between 41-78 years, with a mean age of 56.24 years. Out of 50 patients, 36 (72\%) were males and 14 (28\%) females, with a male to female ratio of 2.6:1. In a study conducted by G. I. Parameswaran et al, there were 57 patients aged between 41-84 years with a mean age of 63 years, out of 
which 27 (47\%) were males and 30 (53\%) females (12).

In our study, the duration of DM varied between one year to 21 years, with a mean duration of 9.45 years, while in the above-quoted study (12) it varied from one year to 39 years, with a mean duration of nine years.

In their study on asymptomatic LEAD patients with T2DM, G. I. Parameswaran et al (12) compared the accuracy of pulse oximetry and ankle-brachial index (ABI) with Doppler waveform analysis for detecting LEAD. They discovered that the pulse oximeter had a sensitivity of $77 \%$, a specificity of $97 \%$, a positive likelihood ratio of $30(95 \% \mathrm{Cl})$ and a negative likelihood ratio of $0.23(95 \% \mathrm{Cl})$, while $\mathrm{ABI}$ had a sensitivity of $63 \%$, a specificity of $97 \%$, a positive likelihood ratio of $24.8(95 \% \mathrm{Cl})$ and a negative like hood ratio of $0.38(95 \% \mathrm{Cl})$. The quoted authors concluded that toe pulse oximetry had a similar accuracy to that of $A B I$ in screening for LEAD in T2DM patients.

In a study on 49 patients (98 limbs) regarding the utility of digital pulse oximetry in the screening of peripheral vascular disease (PVD), JungNan Kwon, Whan-Bong Lee (13) found that DPO had a sensitivity of $87.06 \%$, a specificity of $87.8 \%$, a PPV of $84.3 \%$ and a NPV of $90 \%$, while ABI had a sensitivity of $55.09 \%$, a specificity of 94\%, a PPV of $96.7 \%$ and a NPV of $32.02 \%$; they concluded that digital pulse oximetry could be useful for non-invasive screening devices and $\mathrm{ABI}$ for PVD.
In our study, pocket pulse oximetry has shown a sensitivity of $72 \%$, a specificity of $98 \%$, a PPV of $95 \%$ and a NPV of $90 \%$, while digital pulse oximetry had a sensitivity of $83 \%$, a specificity of $98 \%$, a PPV of $96 \%$ and a NPV of $93 \%$. The results of pocket pulse oximetry and those of digital pulse oxymetry are closely comparable to findings reported by in G. I. Parameswaran et al and Jung-Nam Kwon et al, respectively $(12,13)$.

Ankle-brachial index is considered a standard method for screening of LEAD in practice, but measuring the same is cumbersome since it is time-consuming, needs a dedicated device, and requires technical skills to record it - therefore, not commonly practicable in simple outpatient clinical settings. Furthermore, as reported by previous research, arteries of diabetic patients become rigid and non-compressible due to tunica medium calcification (Monckeberg sclerosis), which results in an erroneously high $\mathrm{ABI}$. Hence, doubts have been cast about the accuracy of the $A B I$ in screening for LEAD among people with diabetes (9).

Many of the previous studies evaluated the accuracy of $\mathrm{ABI}$ along with pulse oximetry in screening of LEAD in diabetic individuals. In contrast to other studies, ours compares the effectiveness of pocket pulse oximetry and digital pulse oximetry findings with a reference Doppler waveform analysis in individuals with no symptoms of LEAD. In our study, ABI is not tested simultaneously with pulse oximetry in the same set of patients. However, a comparative analysis

\begin{tabular}{|l|l|l|l|l|}
\hline Test results & \multicolumn{2}{|l|}{ Pulse oximetry } & \multicolumn{2}{l|}{ Digital pulse oximetry } \\
\cline { 2 - 5 } & Present study & $\begin{array}{l}\text { Parameswaran } \\
\text { et al (12) }\end{array}$ & Present study & $\begin{array}{l}\text { Jung-Nan Kwon } \\
\text { et al (13) }\end{array}$ \\
\hline Sensitivity & $72 \%$ & $77 \%$ & $83 \%$ & $87.06 \%$ \\
\hline Specificity & $98 \%$ & $97 \%$ & $98 \%$ & $87.80 \%$ \\
\hline
\end{tabular}

TAB LE 6. Comparative analysis between the present study and Parameswaran et al's one (12)

TAB LE 7. Comparative analysis of pulse oximetry in the present study and ankle-brachial index results of Parameshwaran et al (12) and Jung-nan Kwon et al (13)

\begin{tabular}{|c|c|c|c|c|}
\hline $\begin{array}{l}\text { Test } \\
\text { results }\end{array}$ & $\begin{array}{l}\text { Pocket pulse } \\
\text { oximetry results of } \\
\text { the present study }\end{array}$ & $\begin{array}{l}\text { Digital pulse } \\
\text { oximetry results of } \\
\text { the present study }\end{array}$ & $\begin{array}{l}\mathrm{ABI}^{*} \text { in } \\
\text { Parameswaran } \\
\text { et al (12) }\end{array}$ & $\begin{array}{l}\text { ABI in } \\
\text { Jung-Nan Kwon } \\
\text { et al (13) }\end{array}$ \\
\hline Sensitivity & $72 \%$ & $83 \%$ & $63 \%$ & $55.09 \%$ \\
\hline Specificity & $98 \%$ & $98 \%$ & $97 \%$ & $94.00 \%$ \\
\hline
\end{tabular}

*ABI=ankle-brachial index 
between $\mathrm{ABI}$ results in the above studies and in our research shows the following findings.

\section{CONCLUSION}

Dulse oximetry may be an additional technique for patients with DM to be screened for LEAD. The procedure has a good sensitivity and specificity in detecting LEAD even in the asymptomatic phase, especially in T2DM patients. When comparing the two types of oximeters, digital pulse oximetry showed a sensitivity of $83 \%$, a specificity of $98 \%$, a PPV of $96 \%$ and a NPV of $93 \%$. In a screening test, the higher the sensitivity, the better.

As a clinical suggestion, all patients with asymptomatic T2DM should undergo the screening tests routinely every six months to detect LEAD.

Conflict of interest: none declared. Financial support: self-funded.

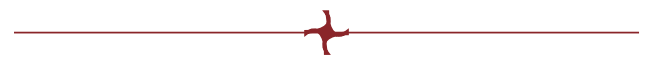

\section{R}

1. Goldstein JA, Mishkel G. Choosing the correct therapeutic option for acute limb ischemia.

Interventional Cardiology 2011;3:381-389.

2. Hardman RL, Jazaeri O, Yi J, et al. Overview of Classification Systems in Peripheral Artery Disease. Semin Intervent Radiol 2014;31:378-388.

3. Katwal AB, Dokun AO. Peripheral Arterial Disease in Diabetes: Is There a Role for Genetics? Curr Diab Rep 2011;11:218-225.

4. Olin JW, Sealove BA. Peripheral Artery Disease: Current Insight Into the Disease and Its Diagnosis and Management. Mayo Clin Proc 2010;85:678-692.

5. Selvin E, Marinopoulos S, Berkenblit G. Meta-analysis: Glycosylated hemoglobin and cardiovascular disease in diabetes mellitus. Ann Intern Med 2004;141:421-431.
6. Hooi JD, Kester HE, Stoffers, et al. Incidence of and risk factors for asymptomatic peripheral arterial occlusive disease: a longitudinal study. Am J Epidemiol 2001;153:666-672.

7. Hirsch AT, Criqui MH, Treat-Jacobson D. Peripheral arterial disease detection in primary care. JAMA 2001;286:1317-1324.

8. Gurpreethsingh Wander,

Bishav Mohan. Vascular disorders of the extremities. In: YP Munjal, SK Sharma, editors. API Textbook of MEDICINE. $10^{\text {th }}$ edition, Vol 1, 2015, pp 1015-1022.

9. Badri PR, Badri VR, Sreekantha SSA. Pulse Oximetry As The Potential Screening Tool For Lower Extremity Arterial Disease In Asymptomatic Patients With Diabetes Mellitus. Int J Res Health Sci [Internet] 2014;2:282-289.
10. Ena J, Argente CR, González-Sánchez V, et al. Use of pocket pulse oximeters for detecting peripheral arterial disease in patients with diabetes mellitus. Journal of Diabetes Mellitus 2013;3:79-85.

11. Siao RM, So MJ, Gomez MH. Pulse Oximetry as a Screening Test for Hemodynamically Significant Lower Extremity Peripheral Artery Disease in Adults with Type 2 Diabetes Mellitus. J ASEAN Fed Endocr Soc 2018;33:130-136.

12. Parameswaran GI, Brand K, Dolan J. Asymptomatic lower extremity arterial disease patients with type 2 diabetes, compared pulse oximetry with $\mathrm{ABI}$ as a screening test for detecting LEAD. J Arch Int Med 2005;16:442-446.

13. Jung-Nam Kwon, Whan-Bong Lee. Utility of digital pulse oximetry in the screening of lower extremity arterial disease. J Korean Surg Soc 2012;82:94-100. 